\title{
Decision-Making Visual Aids for Late, Imaging-Guided Endovascular Thrombectomy for Acute Ischemic Stroke
}

\author{
Pouria Moshayedi, ${ }^{a}$ David S. Liebeskind, ${ }^{\text {a }}$ Ashutosh Jadhav, ${ }^{\mathrm{b}}$ Reza Jahan, ${ }^{\mathrm{c}}$ Maarten Lansberg, ${ }^{\mathrm{d}}$ \\ Latisha Sharma, ${ }^{\mathrm{a}}$ Raul G. Nogueira, ${ }^{\mathrm{e}}$ Jeffrey L. Saver ${ }^{\mathrm{a}}$ \\ aDepartment of Neurology and Comprehensive Stroke Center, University of California Los Angeles, Los Angeles, CA, USA \\ ${ }^{b}$ Department of Neurology, University of Pittsburgh Medical Center, Pittsburgh, PA, USA \\ 'Department of Radiology and Comprehensive Stroke Center, University of California Los Angeles, Los Angeles, CA, USA \\ 'Department of Neurology, Stanford University, Stanford, CA, USA \\ 'Department of Neurology, Emory University, Atlanta, GA, USA
}

Background and Purpose Speedy decision-making is important for optimal outcomes from endovascular thrombectomy (EVT) for acute ischemic stroke (AIS). Figural decision aids facilitate rapid review of treatment benefits and harms, but have not yet been developed for late-presenting patients selected for EVT based on multimodal computed tomography or magnetic resonance imaging.

Methods For combined pooled study-level randomized trial (DAWN and DEFUSE 3) data, as well as each trial singly, 100 person-icon arrays (Kuiper-Marshall personographs) were generated showing beneficial and adverse effects of EVT for patients with AIS and large vessel occlusion using automated (algorithmic) and expert-guided joint outcome table specification.

Results Among imaging-selected patients 6 to 24 hours from last known well, for the full 7-category modified Rankin Scale (mRS), EVT had number needed to treat to benefit 1.9 (interquartile range [IQR], 1.9 to 2.1) and number needed to harm 40.0 (IOR, 29.2 to 58.3). Visual displays of treatment effects among 100 patients showed that, with EVT: 52 patients have better disability outcome, including 32 more achieving functional independence (mRS 0 to 2); three patients have worse disability outcome, including one more experiencing severe disability or death (mRS 5 to 6), mediated by symptomatic intracranial hemorrhage and infarct in new territory. Similar features were present in person-icon figures based on a 6-level mRS (levels 5 and 6 combined) rather than 7-level mRS, and based on the DAWN trial alone and DEFUSE 3 trial alone. Conclusions Personograph visual decision aids are now available to rapidly educate patients, family, and healthcare providers regarding benefits and risks of EVT for late-presenting, imaging-selected AIS patients.

Keywords Intervention; Stroke; Thrombectomy
Correspondence: Pouria Moshayedi Department of Neurology and Comprehensive Stroke Center, University of California Los Angeles, Room 1-240, Neurology Education Office, 710 Westwood Plaza, Los Angeles, CA 900958353, USA

Tel: +1-310-825-6681

Fax: +1-310-206-4733

E-mail: pouria@cantab.net https://orcid.org/0000-0003-17134681

Received: December 18, 2019 Revised: June 13, 2020 Accepted: June 22, 2020 


\section{Introduction}

Randomized trials have recently demonstrated benefit of endovascular mechanical thrombectomy in late-presenting patients with acute ischemic stroke (AIS) due to large vessel occlusion (LVO) who have favorable tissue profiles (small ischemic core, large rescuable penumbra) on multimodal brain imaging. Perfusion-core mismatch patient selection between 6 and 16 hours after last known well and clinical-core mismatch patient selection between 6 and 24 hours after last known well identified patients who benefitted substantially from reperfusion despite relatively late presentation. ${ }^{1,2}$ Accordingly, international guidelines have extended the treatment time-window in AIS due to LVO to include select patients with favorable penumbral profiles on brain imaging between 6 and 24 hours after last known well. As a result, systems of acute stroke care are being reorganized and expanded internationally to facilitate endovascular mechanical thrombectomy in late, imaging-selected LVO-AIS patients. ${ }^{3}$ An important component of stroke care systems requiring optimization for late, imaging-selected LVO-AIS patients are processes to inform patients, families, and physicians regarding the benefits and risks of therapy to facilitate treatment decision-making.

Among all late-presenting LVO-AIS patients, multimodal imaging selects those who have had slow or intermediate, rather than fast, infarct progression up to the time of brain scan. As a result, the pace of benefit loss from endovascular thrombectomy (EVT) associated with prolonged care process times is likely to be less swift among late-presenting, imaging-selected LVOAIS patients than among unselected early-presenting LVO-AIS patients. Nonetheless, multicenter cohort studies have shown that, even among AIS-LVO slow and intermediate progressors, ischemic core growth proceeds at substantial rates. ${ }^{4}$ As a result, experts have emphasized that faster start of EVT after qualifying imaging is desirable to maximize patient benefit even in late, imaging selected patients. ${ }^{5}$ After emergency department arrival, immediate actions leave only a short interval for counselling the patient and/or family about endovascular intervention. In this short period, patients and family without medical knowledge or prior experience must decide whether to pursue a major medical procedure that carries both substantial potential benefits and also substantial potential risks. It is therefore critical to rapidly convey information about the potential beneficial and adverse effects on outcome of mechanical thrombectomy performed in the extended time window. Figural displays are powerful tools to help patients, family, and healthcare providers rapidly understand response patterns to therapy. ${ }^{6,7}$
We sought to use the methods for creating rapidly understandable figural displays from ordinal disability outcomes in parallel group trials ${ }^{6,7}$ to develop visual guides for decision-making regarding ordinal outcome of EVT among late-presenting, imaging-guided, ischemic stroke patients based on data from the two salient pivotal randomized trials, DAWN (Clinical Mismatch in the Triage of Wake Up and Late Presenting Strokes Undergoing Neurointervention With Trevo) and DEFUSE 3 (Endovascular Therapy Following Imaging Evaluation for Ischemic Stroke 3).

\section{Methods}

\section{Study approval and data availability}

The authors declare that all supporting data are available within the article and its online supplementary files. The analyses were performed on publicly available, aggregate group outcome data without any analysis of patient-level data and without any access to patient-level identifiers. Accordingly, this study did not require either ethics approval or Institutional Review Board waiver.

\section{Calculating number needed to treat and benefit per hundred}

The pooled 3-month global disability outcome distributions from the thrombectomy and control arms of the two completed pivotal randomized trials of EVT in late-presenting, imagingselected patients, DAWN and DEFUSE 3, were analyzed. ${ }^{1,2}$ The 3-month modified Rankin Scale (mRS) distributions in the two trials were weighted equally in pooling, yielding the outcome proportions shown in Figure 1. We generated 100 person-icon arrays (Kuiper-Marshall personographs) from this disability dis-

DAWN and DEFUSE 3 combined functional outcome

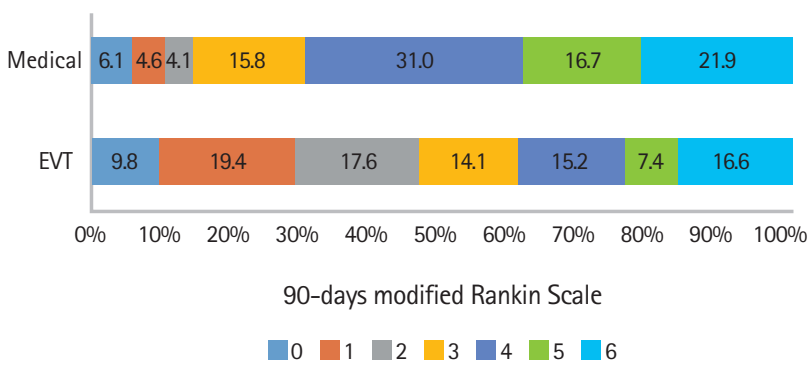

Figure 1. Combined distribution of functional scores on modified Rankin Scale at 90 days. Distributions from DAWN (Clinical Mismatch in the Triage of Wake Up and Late Presenting Strokes Undergoing Neurointervention With Trevo) and DEFUSE 3 (Endovascular Therapy Following Imaging Evaluation for Ischemic Stroke 3) trials were weighted equally. EVT, endovascular thrombectomy. 
tribution displaying treatment outcomes with thrombectomy versus no thrombectomy. ${ }^{12}$

Each visual aid concurrently delineated treatment effects on: (1) increased or decreased degree of 3-month disability, over all seven disability levels of the entire mRS or over a 6-level version of the $m R S$ in which severe disability (mRS 5 ) and death (mRS 6) were combined into a joint worst outcome category; (2) increased functional independence (mRS 0-2); (3) increased severe disability or death (mRS 5-6); and (4) increased symptomatic intracranial hemorrhage $(\mathrm{SICH})$ and infarct in new territory (INT). In addition to the full 7-level mRS analysis, the 6-level $\mathrm{mRS}$ analysis was performed since an $\mathrm{mRS}$ of 5 (severe disability, bedridden, incontinent, and requiring constant nursing care and attention) is not believed to be a better outcome than an mRS of 6 (dead) by nearly half of the individuals at risk. ${ }^{8}$

Absolute risk reductions and increases in the pooled analysis were used to characterize the dichotomized outcomes (mRS 0-2 vs. mRS 3-6) and the SICH binary outcome, benefit per hundred (BPH) and harm per hundred ( $\mathrm{HPH}$ ) values as a result of intervention. For the INT binary outcome, data was available only from DAWN, so HPH was derived from the absolute risk increase observed in that trial.

For the ordinal outcomes, BPH and HPH were defined as the proportion of patients with 3-month disability outcome reduced or increased, respectively, by $\geq 1$ steps on the mRS following therapy. Expert population of joint outcome tables were then used to derive ordinal BPH, HPH, and net BPH. An automated, expert-independent technique, algorithmic min-max population of the joint outcome table, was utilized to calculate ordinal net $\mathrm{BPH} .{ }^{7,910}$

In order to generate the expert joint outcome table method, seven content experts, including noninvasive vascular neurologists (4) and neuro-interventionalists (3) independently populated joint outcome tables. All participants were members of academic institutions who had extensive research and clinical experience in neurothrombectomy. In a joint outcome table display of 1,000 patients, each patient specified the individual patient responses to treatment that would match observed trial results. Each expert was instructed to choose the array of individual patient responses that, in their judgment, was the most biologically possible clinical response, constrained by the requirement that the distribution be consistent with group-level pooled results. While completing the arrays, in addition to the group-level mRS outcomes, the rates of SICH and INT derived from DAWN and DEFUSE 3 studies were provided to experts.

In addition to the main visual aid derivation, based on the pooled DAWN and DEFUSE 3 results, we also performed ex- planatory visual aid derivations, based on DAWN alone and DEFUSE 3 alone. For derivation of these trial-specific personographs, separate joint outcome tables were constructed showing: (1) in DAWN, the mRS outcome distributions across all seven levels, the rate of $\mathrm{SICH}$, and the rate of INT; and (2) in DEFUSE 3, the mRS outcome distributions across all seven levels and the rate of SICH (with the rate of INT imputed from DAWN as it was not reported for DEFUSE 3). One expert rater highly experienced with joint outcome table population (Jeffrey L. Saver) then separately populated the DAWN joint outcome table and the DEFUSE 3 joint outcome table. That expert rater's Joint Outcome Table (JOT) population of the combined DAWN-DEFUSE 3 data had yielded number needed to treat to benefit (NNTB) and number needed to treat to harm (NNTH) values that were in middle of the range of all seven raters.

\section{Person-icon figures generation}

Kuiper-Marshall personographs were used as the visual aid displays to depict the benefit and harm of the thrombectomy intervention in imaging-selected, delayed time-window patients. Two types of lead visual decision aids were generated, based on the 7-level mRS and the pooled DAWN-DEFUSE 3 data: (1) a Single Personograph Choice Consequence Matrix display; and (2) a Double Personograph Choice Comparison display. In addition, Single Personograph Choice Consequence Matrix displays were developed for: (1) the 6-level mRS for the pooled DAWNDEFUSE 3 data; (2) the 7-level mRS using DAWN data alone; and (3) the 7-level mRS using DEFUSE 3 data alone.

Choice Consequence Matrix visual decision aids take the outcomes of patients undergoing control therapy as a given and depict the shifts in these outcomes that occur as a result of interventional treatment. Choice Consequence Matrix personographs were generated using the median BPH and HPH values from the expert joint outcome tables to show changes in outcome expected among 100 treated patients, as a result of treatment. For the 100 person-icon array, three-color shadings were used to depict three-wide changes in long-term, 3-month disability outcomes among 100 treated patients following thrombectomy versus no thrombectomy: (1) having a less disabled outcome with thrombectomy than no thrombectomy, by $\geq 1$ levels on the mRS (green color); (2) having no changes in 3-month disability outcome (white color); and (3) having a more disabled outcome with thrombectomy than no thrombectomy, by $\geq 1$ levels on the mRS (red color). In order to show the proportion of patients improving to functional independence the green icons were further differentiated: mRS 0 to 2, as a result of treatment (dark green) and improving to lesser levels of disability (light green). The red icons were also 
differentiated to show the proportion of patients worsening to severe disability or death, mRS 5 to 6 , as a result of treatment (dark red) and worsening to lesser levels of disability (light red). In order to depict the two short-term outcomes of SICH and INT closed dashes and open dashes were added, respectively.

For some physician, severe disability (mRS 5) is not significantly different from death (mRS 6). To enable physicians communicate alternative benefit-harm aspect perspectives with patients, an additional choice consequence figure have been developed considering the 6-level, rather than 7-level version, in which of severe disability (mRS 5) and death (mRS 6) are combined into a single worst possible outcome category.

Choice Comparison visual decision aids show, side-by-side, patient outcomes under each treatment option. The Choice Comparison visual display was generated by showing two adjacent 100 person-icon arrays depicting: 90-day mRS outcomes with thrombectomy and 90-day mRS outcomes with medical treatment. The group distribution of the $7 \mathrm{mRS}$ levels in the pooled DAWN and DEFUSE 3 analysis was used to assign each person-icon an mRS level. A spectrum of color gradients was employed to communicate the degree of disability associated with each level of the mRS: $m R S 0$, dark green; $m R S 1$, medium green; $m R S 2$, light green; $m R S 3$, yellow; $m R S 4$, light red; $m R S 5$, medium red; $m R S$ 6, dark red. Therefore mRS outcomes were shown with both broad and granular distinctions: green color was used to broadly identify favorable outcomes (and further differentiated by intensity of green shading), yellow color broadly showed intermediate outcomes, and red color broadly marked unfavorable outcomes (and further differentiated by intensity of red shading).

\section{Results}

In DAWN there were 206 patients, 107 assigned to the thrombectomy group and 99 to the control group, and in DEFUSE 3 there were 182 patients, 92 assigned to the thrombectomy group and 90 to the control group. The study-level averaged distributions of the 3-month mRS with EVT and with control is shown in Figure 1. The mean mRS score in the EVT group was 2.94 (standard deviation [SD], 1.94) and in the medical group 3.98 (SD, 1.67), yielding a mean difference of 1.04. The studylevel averaged rates of $\mathrm{SICH}$ were $6.1 \%$ with EVT and 3.7\% with medical therapy and the rate of INT, available from DAWN, was $3.7 \%$ with EVT and $0 \%$ with medical therapy. For each of the six possible dichotomizations of the 7-level mRS, net BPH values are shown in Table 1 and ranged from 4 to 32 .

The independent expert joint outcome analyses indicated that the biologically most plausible $\mathrm{BPH}$, of patients having a
Table 1. BPH and HPH values for endovascular thrombectomy in late-presenting, imaging-selected patients

\begin{tabular}{|c|c|c|c|}
\hline Variable & $\mathrm{BPH}$ & $\mathrm{HPH}$ & Net BPH \\
\hline \multicolumn{4}{|c|}{ For transitions across all 7 levels of mRS } \\
\hline Biologically most plausible* & 52.1 & 2.5 & 47.6 \\
\hline Automatically calculated $^{+}$ & & & 53.0 \\
\hline \multicolumn{4}{|c|}{ For individualdichotomizations of $\mathrm{mRS}^{\ddagger}$} \\
\hline 0 vs. $1-6$ & & & 4 \\
\hline $0-1$ vs. $2-6$ & & & 19 \\
\hline $0-2$ vs. $3-6$ & & & 32 \\
\hline $0-3$ vs. $4-6$ & & & 30 \\
\hline $0-4$ vs. $5-6$ & & & 15 \\
\hline $0-5$ vs. 6 & & & 5 \\
\hline
\end{tabular}

$\mathrm{BPH}$, benefit per hundred; $\mathrm{HPH}$, harm per hundred; $\mathrm{mRS}$, modified Rankin Scale.

*Via expert population of joint outcome table; ${ }^{\top}$ Via algorithmic min-max population of joint outcome table; ${ }^{\dagger} \mathrm{V}$ ia absolute risk difference.

better outcome with thrombectomy by $\geq 1$ levels on the mRS, was 52.1 (interquartile range [IQR], 48.2 to 52.6 ) and the $\mathrm{HPH}$ 2.5 (IQR, 1.8 to 3.5). The likelihood of help to harm ratio was 20.8. The net BPH from the expert JOT (eJOT) analysis was 47.6 (IQR, 45.4 to 49.6), comparable to the automatically derived net BPH of 53.0. The NNTB was 1.9 (IQR, 1.9 to 2.1), and NNTH was 40.0 (IQR, 29.2 to 58.3).

Combining the eJOT full mRS shift and the BPH analysis indicated that among 100 patients treated with thrombectomy 52 have a reduced final level of disability as a result of treatment, including 32 who improve to an independent functional outcome (mRS 0-2) and 20 whose final disability reduction occurs across other transitions on the mRS besides the mRS 3 to 2 transition. The eJOT HPH analysis also indicated that among these 100 treated patients, three would have an increased final level of disability as a result of thrombectomy, including one who worsens to a severely disabled or dead (mRS 5-6) outcome and two whose final disability worsening occurs across other transitions on the mRS besides the mRS 4 to 5 transition. These values were used to generate the lead decision matrix visual decision aid for late-presenting imaging-selected patients treated with thrombectomy (Figure 2). In a similar manner to above analyses, three additional Choice Consequence Matrix type visual decision aids were: (1) 7-level $m R S$ analysis using DAWN data only (Figure 3A); (2) 7-level mRS analysis using DEFUSE 3 data only (Figure 3B); and (3) 6-level mRS analysis using pooled DAWN-DEFUSE 3 (Supplementary Figure 1) and DAWN or DEFUSE 3 individually (Supplementary Figure 2).

The Choice Comparison visual decision aid using seven color 
Thrombectomy for late, imaging selected AIS due to large vessel occlusion (based on 7-level mRS disability scale)
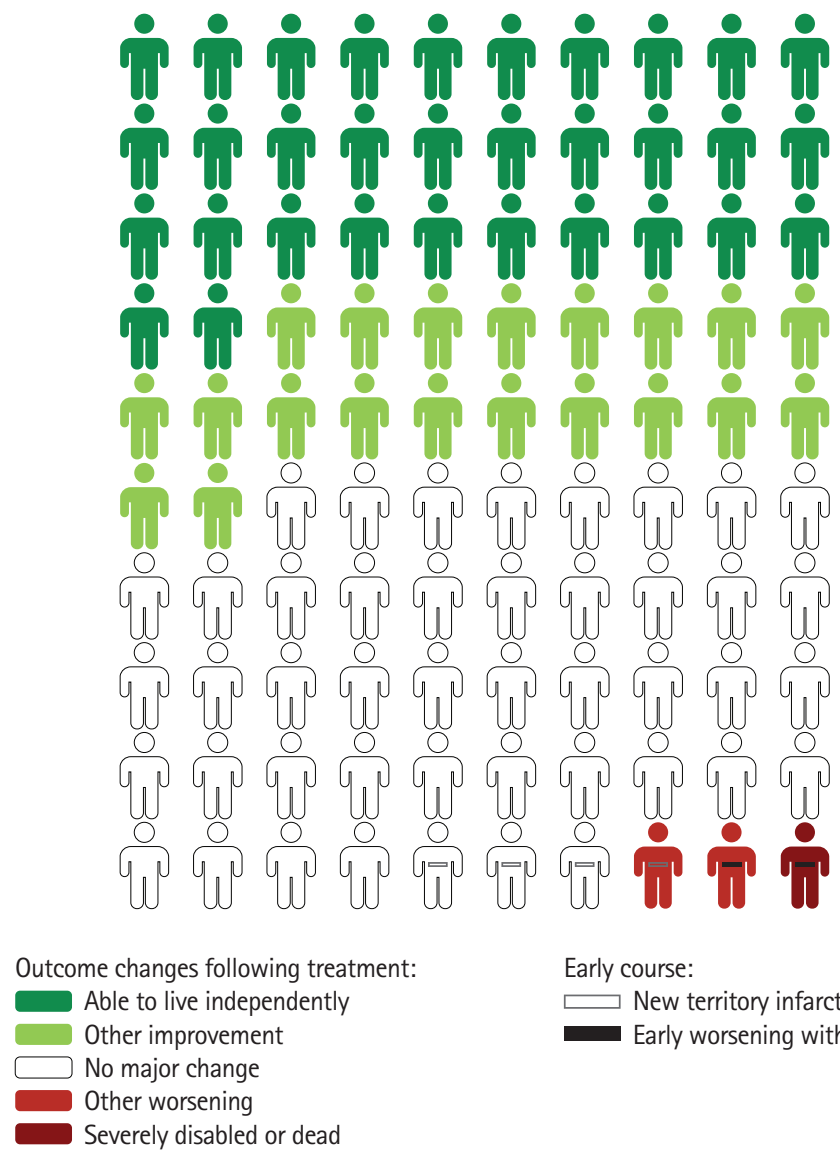

Early course:

$\square$ New territory infarct

Early worsening with brain bleeding

Severely disabled or dead

Figure 2. Choice Consequence Matrix type visual decision aid depicting the benefits and risks of endovascular thrombectomy (EVT) in late-presenting, imaging-selected patients. Colors indicate changes in 3-month level of global disability that occur as a result of EVT, considering all 7 levels of the modified Rankin Scale (mRS). Dark green, attainment of functional independence (mRS 0-2) as a result of thrombectomy; light green, improved disability outcome (other than functional independence) as a result of thrombectomy; dark red, severely disabled or dead outcome (mRS 5-6) as a result of thrombectomy; light red, worse disability outcome (other than severely disabled/dead) as a result of thrombectomy; closed dash, symptomatic intracranial hemorrhage as a result of thrombectomy; and open dash, new territory infarct as a result of thrombectomy. Figure freely available under a Creative Commons 4.0, use freely with attribution license. Personographs based on outcomes among patients enrolled in the DAWN (Clinical Mismatch in the Triage of Wake Up and Late Presenting Strokes Undergoing Neurointervention With Trevo) and DEFUSE 3 (Endovascular Therapy Following Imaging Evaluation for Ischemic Stroke 3) randomized trials. Imaging selection strategies in those trials were: DAWN (clinical-core mismatch at 6 to 24 hours, indicated by: age $\geq 80$, National Institutes of Health Stroke Scale [NIHSS] $\geq 10$, and ischemic core $<21 \mathrm{~mL}$; age $<80$, NIHSS $\geq 10$, and ischemic core $<31 \mathrm{~mL}$; or age $<80$, NIHSS $\geq 20$, and ischemic core $<51 \mathrm{~mL}$ ); DEFUSE 3 (perfusion-diffusion mismatch at 6 to 16 hours, indicated by: ischemic core $<70 \mathrm{~mL}$, ratio of perfusion to core volumes 1.8 , and penumbra volume $\geq 15 \mathrm{~mL}$ ). AlS, acute ischemic stroke.

shades to depict all 3-month mRS outcomes in both EVT and medically treated patients is shown in the Supplementary Figure 3.

\section{Discussion}

In the present study, visual aids have been produced to convey key decisional information to patients, families, and providers regarding benefits and harms of EVT in late-presenting, imaging-selected patients, encompassing benefits and harms over all levels of 3-month disability, as well as early adverse effects of SICH and INT. Expert-informed derivation of benefits and harms per hundred treated patients considering disability tran- sitions across all seven levels of the mRS and automated, mathematical derivations demonstrated good agreement. In the expert-assisted derivation, among every 100 patients treated with EVT, 52 will have an improved and three a worse 3-month global disability level. Clinicians can use the developed visual decision aids to rapidly inform patients and family members of the benefits and risks associated with EVT for AIS in penumbral-imaging selected patients 6 to 24 hours after last known well.

The BPH and HPH values for shift in 3-month mRS outcomes derived in the current study, and depicted in the resulting visual aids, are consonant with, and extend, prior related findings. 
Thrombectomy for late, imaging selected AIS due to large vessel occlusion: DAWN Trial (based on 7-level mRS disability scale)

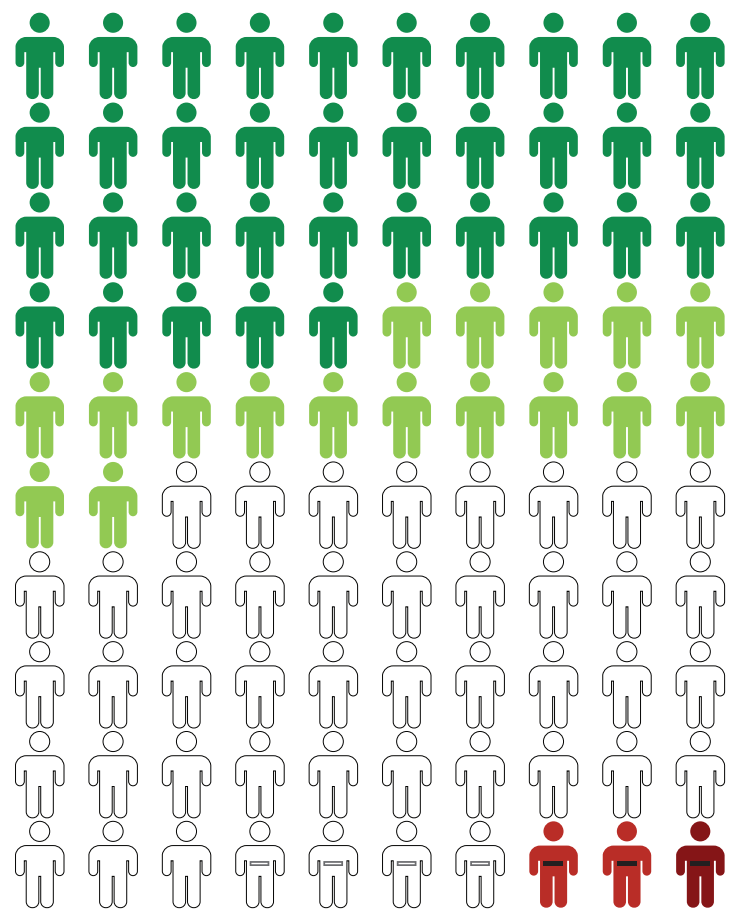

Outcome changes following treatment:

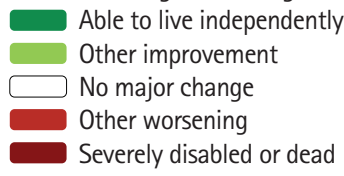

Thrombectomy for late, imaging selected AIS due to large vessel occlusion: DEFUSE 3 Trial (based on 7-level mRS disability scale)

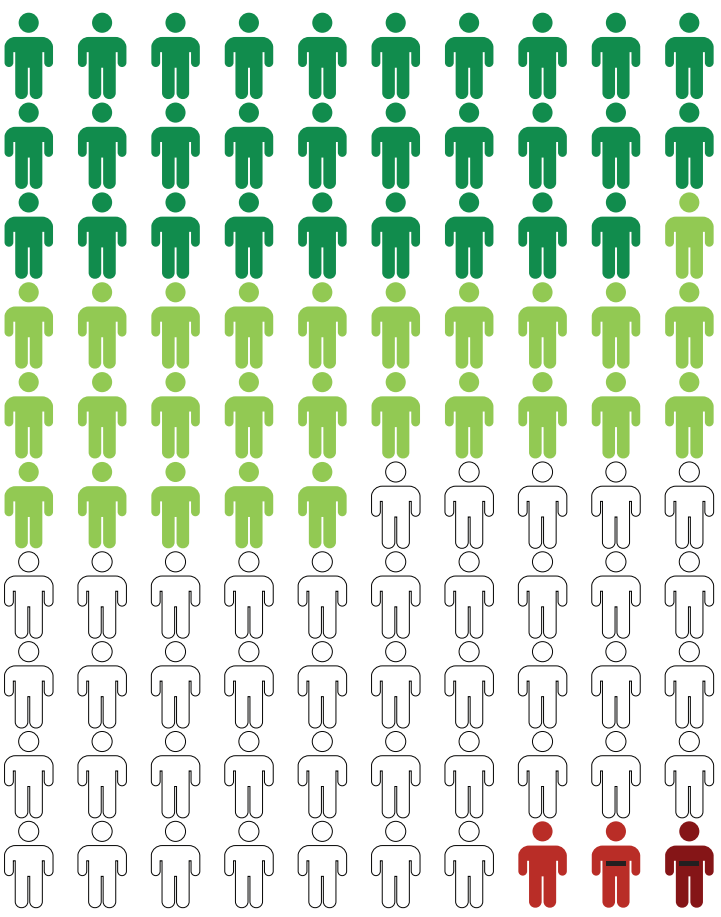

Early course:

$\square$ New territory infarct

Early worsening with brain bleeding

Figure 3. Choice Consequence Catrix type visual decision aids depicting the benefits and risks of endovascular thrombectomy (EVT) in late-presenting, imaging-selected patients in the DAWN (Clinical Mismatch in the Triage of Wake Up and Late Presenting Strokes Undergoing Neurointervention With Trevo) trial (A) and the DEFUSE 3 (Endovascular Therapy Following Imaging Evaluation for Ischemic Stroke 3) trial (B). Colors indicate changes in 3-month level of global disability that occur as a result of EVT, considering all seven levels of the modified Rankin Scale (mRS). Dark green, attainment of functional independence (mRS 0-2) as a result of thrombectomy; light green, improved disability outcome (other than functional independence) as a result of thrombectomy; dark red, severely disabled or dead outcome (mRS 5-6) as a result of thrombectomy; light red, worse disability outcome (other than severely disabled/dead) as a result of thrombectomy; closed dash, symptomatic intracranial hemorrhage as a result of thrombectomy; and open dash, new territory infarct as a result of thrombectomy (only reported for DAWN trial). Figures freely available under a Creative Commons 4.0, use freely with attribution license. AIS, acute ischemic stroke.

The magnitude of EVT shift effects in the late-presenting, imaging-selected EVT patients is comparable to the effects of early EVT in largely under 6-hour patients ineligible for intravenous thrombolytic therapy, and the net benefits substantially exceed that of intravenous thrombolysis alone in under 3-hour patients. ${ }^{6,7}$ This accords with the greater reperfusion efficacy of EVT over intravenous thrombolysis and with the capacity of multimodal imaging to identify those late-presenting patients who are similar to the preponderance of early-presenting patients in still harboring substantial salvageable penumbra.

Producing visual decision aids delineating dichotomized end points is relatively straightforward, but figural displays are more challenging to develop for ordinal outcomes that are ar- rayed along a range of disability. Fortunately, techniques to produce rapidly understandable figural displays from ordinal disability outcomes in parallel group trials have been developed. ${ }^{6,7}$ Though, to our knowledge, expert derivation of differentiated BPH and HPH for EVT in imaging-selected, late-presenting patients has not been previously performed, our findings are consistent with an automated derivation of net BPH performed on the DAWN trial dataset using a joint outcome table multiple resampling technique. ${ }^{2}$ The net BPH value for DAWN alone of 50 in that study is comparable to the net BPH values for pooled DAWN-DEFUSE 3 data in the current study using the expert joint outcome table population technique (net BPH 48) and the automated, algorithmic min-max, joint out- 
come table population technique (net BPH 53).

The general comparability in the current study of the net BPH derived by the expert-informed and expert-independent methods strengthens confidence in the results and provides assurance that the expert judgments are reliable. That the expert-derived net BPH was actually mildly lower than the algorithmically derived net BPH further indicates that the experts did not "game" their joint outcome table completions to inflate the BPH. Rather, as a result of their content expertise, they judged that those patients who benefit from EVT benefit substantially, so that the group-level benefit of EVT observed in overall trial results is distributed to fewer patients than indicated by the automated algorithmic technique.

The visual decision aids developed in this study meet key recommendations of the International Patient Decision Aids Collaboration for developing effective decision aids for emergency treatments. ${ }^{6,11}$ These include (1) figures are based on input from disease-specific experts and experts in multiple related specialties; (2) figures are generated according to the highest available quality of research data; (3) an appropriate graph type is chosen for the type of information to be conveyed (in this instance, Kuiper-Marshall personographs for betweengroup outcome comparison); (4) information includes both benefits and risks associated with a treatment option; (5) the elements of the graphical display are shown proportional to the data quantiles; (6) absolute indices rather than relative outcomes are displayed; (7) a common denominator is used to compare outcome probabilities in different treatment options; and (8) color and symbol formatting is aligned with the degree to which outcomes are important and meaningful for patients. In the lead visual decision aid display format (Single Personograph Choice Consequence Matrix), large person-icons convey the most important outcome, 3-month level of disability, using color-coding to distinguish five effect levels (improvement to functional independence, improvement though not to functional independence, no alteration, worsening though not to severe disability-death, and worsening to severe disabilitydeath). In addition, the less important (because short-term) outcomes of early SICH and imaged INT are conveyed by smaller bars placed within appropriate person-icons. In the alternative visual decision aid display format (Double Personograph Choice Comparison), person-icons convey the most important outcome, 3-month level of disability, using color-coding to distinguish all seven levels of the mRS, showing the expected outcome distributions with EVT+medical management vs. medical management alone side-by-side. Physicians, nurses, patients, and families vary in their preference regarding whether to use Choice Consequence Matrix or Choice Comparison vi- sual displays for decision-making, so both have been generated. But most end-users prefer to use the Choice Consequence Matrix display as focuses viewer attention solely upon changes in outcome directly attributable to thrombectomy treatment, leaving aside other influences upon outcome that may be more challenging for patients and families to process quickly. The Choice Consequence Matrix display was therefore developed as the lead display in this study.

A caution to note is that, while both the DAWN and DEFUSE 3 trials used advanced imaging techniques for patient selection among late-presenting patients, some technical aspects of the imaging-selection strategies differed between the two trials. Both trials used advanced imaging to identify patients with favorable penumbral profiles, characterized by large volumes of threatened but still salvageable tissue (penumbra) and small volumes of likely irreversibly-infarcted tissue (ischemic core). 1,2,12-14 DEFUSE 3 used a perfusion-core mismatch imaging strategy to identify favorable penumbral profile patients. DAWN permitted sites to either also use the perfusion-core mismatch strategy or, at magnetic resonance imaging (MRI) sites able to perform diffusion but not perfusion MRI, to use a clinical-core mismatch strategy identify favorable penumbral profile patients. As both trials' imaging strategies select for similar small core/large penumbra patients, it would be pathophysiologically expected that they would be enrolling very similar patients, and formal studies have empirically confirmed this similarity by showing that patients selected by both imaging strategies respond to EVT similarly. ${ }^{12,15}$

The findings of the current study provide further support that the DAWN and DEFUSE 3 imaging strategies select largely similar patients. The BPH values for the DAWN trial alone (52) and the BPH values for the DEFUSE 3 trial alone (55) were very similar, as were the HPH values for the DAWN trial alone (3) and for the DEFUSE 3 trial alone (3). As a result, the Choice Consequence Matrix visual decision aids for each trial alone were very similar in appearance.

An additional caution is that the visual aids generated in this study show outcomes and outcome differences expected for a prototypical patient of the type enrolled in the DAWN and DEFUSE 3 trials, and each individual patient in front of a clinician at time of acute treatment decision-making is likely to differ in several features from the prototypical trial patient. Every individual patient has particular prognostic features, including age, admission National Institutes of Health Stroke Scale (NIHSS) score, time from last known well, and imaged infarct core size, that differentiate them somewhat from the overall trial population and will shape their specific outcomes with EVT and with medical therapy alone. ${ }^{16}$ Nonetheless, fixed decision aid 
images that reflect a prototypical patient enrolled in trials with largely similar populations can be useful in emergency settings where rapid access to the decision aid is a requisite. For this reason, fixed decision aid images for prototypical patients merging data from several acute stroke studies have found wide adoption for intravenous thrombolysis and EVT therapy for AIS. ${ }^{7,17,18}$ Personalized, patient-specific visual decision aids able to be generated in real-time after input of individual patient characteristics have been developed for thrombolytic stroke therapy and other cardiovascular treatments, but have thus far been confined to presentation of binary dichotomized outcomes, and so substantially underestimate the full benefits and full harms of therapy. ${ }^{19,20}$

This study has additional limitations. First, while data regarding 3-month global disability early SICH outcomes were available from both trials, data regarding infarcts in new territories was available only from DAWN. Second, the generated figures reflect outcomes of EVT when performed by experienced neuro-interventionalists during the time period of conduct of the DAWN and DEFUSE 3 trials. The benefits of EVT may not be as great when performed at centers with less experienced interventionalists and supporting services; conversely, the benefits of EVT may be heightened when performed with technologies that have become available only after the completion of the DAWN and DEFUSE 3 trials. ${ }^{21}$ Third, the DAWN and DEFUSE 3 trials each recruited patients with extremely favorable patterns on advanced imaging. The substantial net benefit from EVT in these trials suggests that patients with mildly less favorable imaging profiles (modestly larger ischemic core volume and/or modestly smaller penumbra volume) are still likely to benefit meaningfully from EVT. But the current figures are not applicable to such patients who do not quite formally meet either DAWN or DEFUSE 3 entry criteria. Lastly it is important to emphasize that direct patient-practitioner and/or family-practitioner discussions are pivotal for optimal treatment decisionmaking; the visual aids generated in the current study are intended to facilitate, not substitute for, those discussions.

\section{Conclusions}

Mechanical thrombectomy in late-presenting, imaging-selected patients with ischemic stroke due to LVO confers substantial benefit, reducing long term disability across the entire mRS range in 52\% of treated patients, and minimal harm, increasing long term disability in 3\% of patients. Figural decision guides are now available that amalgamate in one rapidly assimilable image, long term benefits, long term harms, and short term complications. These visual displays can convey the most salient information necessary for decision making rapidly and efficiently in emergency settings. The figures may also be helpful in educating policy-makers, the general public, and key stakeholders designing regional systems of acute stroke care regarding the magnitude of benefit and harm of EVT in latepresenting, imaging-selected stroke patients.

\section{Supplementary materials}

Supplementary materials related to this article can be found online at https://doi.org/10.5853/jos.2019.03503.

\section{Disclosure}

Pouria Moshayedi is an employee of the University of California.

David S. Liebeskind is an employee of the University of California. David S. Liebeskind is consultant to Cerenovus, Genentech, Stryker and Medtronic as Imaging Core Lab.

Ashutosh Jadhav declared no relevant conflict of interests.

Reza Jahan is an employee of the University of California. Reza Jahan reports being a consultant for Medtronic Neurovascular, Balt USA, BlackSwan Vascular Inc., and Viz.ai outside the submitted work.

Maarten Lansberg declared no relevant conflict of interest.

Latisha Sharma is an employee of the University of California.

Raul G. Nogueira has declared the following interests: Stryker Neurovascular (DAWN Trial Principal Investigator, no compensation; TREVO Registry Steering Committee, no compensation; Trevo-2 Trial Principal Investigator, modest; Consultant, significant); Medtronic (SWIFT Trial Steering Committee, modest; SWIFT-Prime Trial Steering Committee, no compensation; STAR Trial Angiographic Core Lab, significant); Penumbra (3D Separator Trial Executive Committee, no compensation); Cerenovus/Neuravi (ENDOLOW Trial Principal Investigator, EXCELLENT Registry Principal Investigator, ARISE-2 trial Steering Committee, no compensation; Physician Advisory Board, modest); Phenox (PROST Trial Principal Investigator, Physician Advisory Board: modest); Anaconda (Physician Advisory Board, modest); Genentech (Physician Advisory Board, modest); Biogen (CHARM Trial Steering Committee; Physician Advisory Board, modest); Prolong Pharmaceuticals (Physician Advisory Board, modest); Allm Inc. (Physician Advisory Board, no compensation); IschemaView (Speaker, modest); Brainomix (Physician Advisory Board, stock options); Sensome (Research Device Use, no compensation); Viz-Al (Physician Advisory Board, stock options); Philips (Research Software Use, no compensation; 
Speaker, modest); Corindus Vascular Robotics (Physician Advisory Board, stock options); Vesalio (Physician Advisory Board, stock options); Ceretrieve (Physician Advisory Board, stock options); Astrocyte (Physician Advisory Board, stock options).

Jeffrey L. Saver is an employee of the University of California. The University of California has patent rights in retrieval devices for stroke. Jeffrey L. Saver has served as an unpaid site investigator in multicenter trials sponsored by Medtronic, Stryker, and Cerenovus for which the UC Regents received payments on the basis of clinical trial contracts for the number of subjects enrolled. Jeffrey L. Saver has received contracted hourly payments from Medtronic, Stryker, and Cerenovus and stock options from Rapid Medical for service on Clinical Trial Steering Committees advising on rigorous trial design and conduct.

\section{References}

1. Albers GW, Marks MP, Kemp S, Christensen S, Tsai JP, OrtegaGutierrez $S$, et al. Thrombectomy for stroke at 6 to 16 hours with selection by perfusion imaging. N Engl J Med 2018;378: 708-718.

2. Nogueira $R G$, Jadhav $A P$, Haussen $D C$, Bonafe $A$, Budzik RF, Bhuva $P$, et al. Thrombectomy 6 to 24 hours after stroke with a mismatch between deficit and infarct. N Engl J Med 2018;378:1121.

3. Mokin $M$, Ansari SA, McTaggart RA, Bulsara KR, Goyal M,

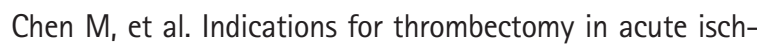
emic stroke from emergent large vessel occlusion (ELVO): report of the SNIS Standards and Guidelines Committee. J Neurointerv Surg 2019;11:215-220.

4. Wheeler HM, Mlynash $M$, Inoue $M$, Tipirnini $A$, Liggins J, Bammer $R$, et al. The growth rate of early DWI lesions is highly variable and associated with penumbral salvage and clinical outcomes following endovascular reperfusion. Int $\mathrm{J}$ Stroke 2015;10:723-729.

5. Hill MD, Goyal M. Treat fast but abandon time from ischemic stroke onset as a criterion for treatment: the DAWN and DEFUSE-3 trials. Int J Stroke 2018;13:344-347.

6. Gadhia J, Starkman S, Ovbiagele B, Ali L, Liebeskind D, Saver $J$ L. Assessment and improvement of figures to visually convey benefit and risk of stroke thrombolysis. Stroke 2010;41:300306.

7. Tokunboh I, Vales Montero M, Zopelaro Almeida MF, Sharma L, Starkman S, Szeder V, et al. Visual aids for patient, family, and physician decision making about endovascular thrombectomy for acute ischemic stroke. Stroke 2018;49:90-97.

8. Samsa GP, Matchar DB, Goldstein L, Bonito A, Duncan PW, Lipscomb J, et al. Utilities for major stroke: results from a survey of preferences among persons at increased risk for stroke. Am Heart J 1998;136(4 Pt 1):703-713.

9. Howard G, Waller JL, Voeks JH, Howard VJ, Jauch EC, Lees $K R$, et al. A simple, assumption-free, and clinically interpretable approach for analysis of modified Rankin outcomes. Stroke 2012;43:664-669.

10. Saver JL, Gornbein J, Grotta J, Liebeskind D, Lutsep H, Schwamm $L$, et al. Number needed to treat to benefit and to harm for intravenous tissue plasminogen activator therapy in the 3- to 4.5-hour window: joint outcome table analysis of the ECASS 3 trial. Stroke 2009;40:2433-2437.

11. Durand MA, Witt J, Joseph-Williams N, Newcombe RG, Politi $M C$, Sivell $S$, et al. Minimum standards for the certification of patient decision support interventions: feasibility and application. Patient Educ Couns 2015;98:462-468.

12. Leslie-Mazwi TM, Hamilton $S$, Mlynash M, Patel $A B$, Schwamm LH, Lansberg MG, et al. DEFUSE 3 non-DAWN patients. Stroke 2019;50:618-625.

13. Bivard A, Huang $X$, Levi CR, Campbell BC, Cheripelli BK, Chen

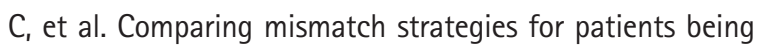
considered for ischemic stroke Tenecteplase trials. Int J Stroke 2020;15:507-515.

14. Powers WJ, Rabinstein AA, Ackerson T, Adeoye OM, Bambakidis NC, Becker K, et al. Guidelines for the early management of patients with acute ischemic stroke: 2019 update to the 2018 guidelines for the early management of acute ischemic stroke: a guideline for healthcare professionals from the American Heart Association/American Stroke Association. Stroke 2019;50:e344-e418.

15. Ducroux C, Khoury N, Lecler A, Blanc R, Chetrit A, Redjem H, et al. Application of the DAWN clinical imaging mismatch and DEFUSE 3 selection criteria: benefit seems similar but restrictive volume cut-offs might omit potential responders. Eur J Neurol 2018;25:1093-1099.

16. Kent DM, Steyerberg E, van Klaveren D. Personalized evidence based medicine: predictive approaches to heterogeneous treatment effects. BMJ 2018;363:k4245.

17. McMeekin $P$, Flynn D, Ford GA, Rodgers H, Gray J, Thomson RG. Development of a decision analytic model to support decision making and risk communication about thrombolytic treatment. BMC Med Inform Decis Mak 2015;15:90.

18. Saposnik G, Goyal M, Majoie C, Dippel D, Roos Y, Demchuk A, et al. Visual aid tool to improve decision making in acute stroke care. Int J Stroke 2016;11:868-873.

19. Decker C, Chhatriwalla E, Gialde E, Garavalia B, Summers $D$, Quinlan ME, et al. Patient-centered decision support in acute ischemic stroke: qualitative study of patients' and providers' perspectives. Circ Cardiovasc Qual Outcomes 2015;8(6 Suppl 
3):S109-S116.

20. Flynn D, Nesbitt DJ, Ford GA, McMeekin P, Rodgers H, Price $C$, et al. Development of a computerised decision aid for thrombolysis in acute stroke care. BMC Med Inform Decis Mak 2015;15:6.
21. Zaidat 00, Bozorgchami $H$, Ribó $M$, Saver JL, Mattle HP, Chapot $R$, et al. Primary results of the multicenter ARISE II study (Analysis of Revascularization in Ischemic Stroke With EmboTrap). Stroke 2018;49:1107-1115. 
Thrombectomy for late, imaging selected AIS due to large vessel occlusion (based on 6-level mRS disability scale)

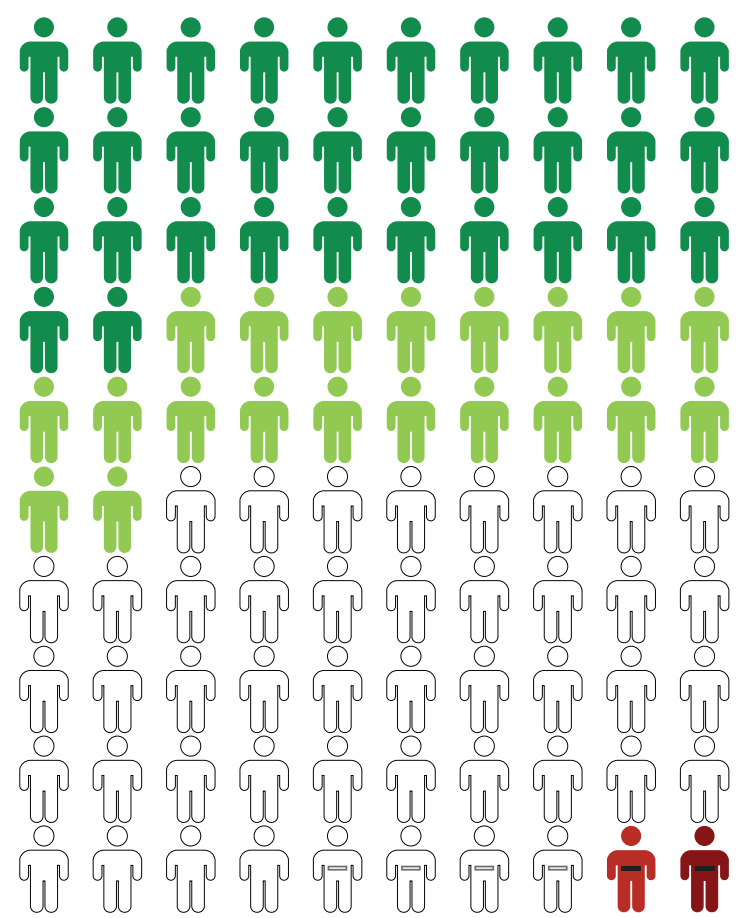

Outcome changes following treatment: Able to live independently Other improvement

No major change

Other worsening

Severely disabled or dead
Early course:

$\square$ New territory infarct

Early worsening with brain bleeding

Supplementary Figure 1. Choice Consequence Matrix type visual decision aid depicting the benefits and risks of endovascular thrombectomy (EVT) in latepresenting, imaging-selected patients. Colors indicate changes in 3-month level of global disability that occur as a result of EVT, considering 6 of the levels of the modified Rankin Scale (mRS) with mRS levels 5 and 6 combined into a single worst outcome level. Dark green, attainment of functional independence (mRS 0-2) as a result of thrombectomy; light green, improved disability outcome (other than excellent outcome) as a result of thrombectomy; dark red, severely disabled, or dead outcome (mRS 5-6) as a result of thrombectomy; light red, worse disability outcome (other than severely disabled/dead) as a result of thrombectomy; closed dash, symptomatic intracranial hemorrhage as a result of thrombectomy; and open dash, new territory infarct as a result of thrombectomy. Figure freely available under a Creative Commons 4.0, use freely with attribution license. Personographs based on outcomes among patients enrolled in the DAWN (Clinical Mismatch in the Triage of Wake Up and Late Presenting Strokes Undergoing Neurointervention With Trevo) and DEFUSE 3 (Endovascular Therapy Following Imaging Evaluation for Ischemic Stroke 3) randomized trials. Imaging selection strategies in those trials were: DAWN (clinical-core mismatch at 6 to 24 hours, indicated by: age $\geq 80$, National Institutes of Health Stroke Scale [NIHSS] $\geq 10$, and ischemic core $<21 \mathrm{~mL}$; age $<80$, NIHSS $\geq 10$, and ischemic core $<31 \mathrm{~mL}$; or age $<80$, NIHSS $\geq 20$, and ischemic core $<51 \mathrm{~mL}$ ); DEFUSE 3 (perfusion-diffusion mismatch at 6 to 16 hours, indicated by: ischemic core $<70 \mathrm{~mL}$, ratio of perfusion to core volumes 1.8 , and penumbra volume $\geq 15 \mathrm{~mL}$ ). AlS, acute ischemic stroke. 
Thrombectomy for late, imaging selected AIS due to large vessel occlusion: DAWN Trial (based on 6-level mRS disability scale)

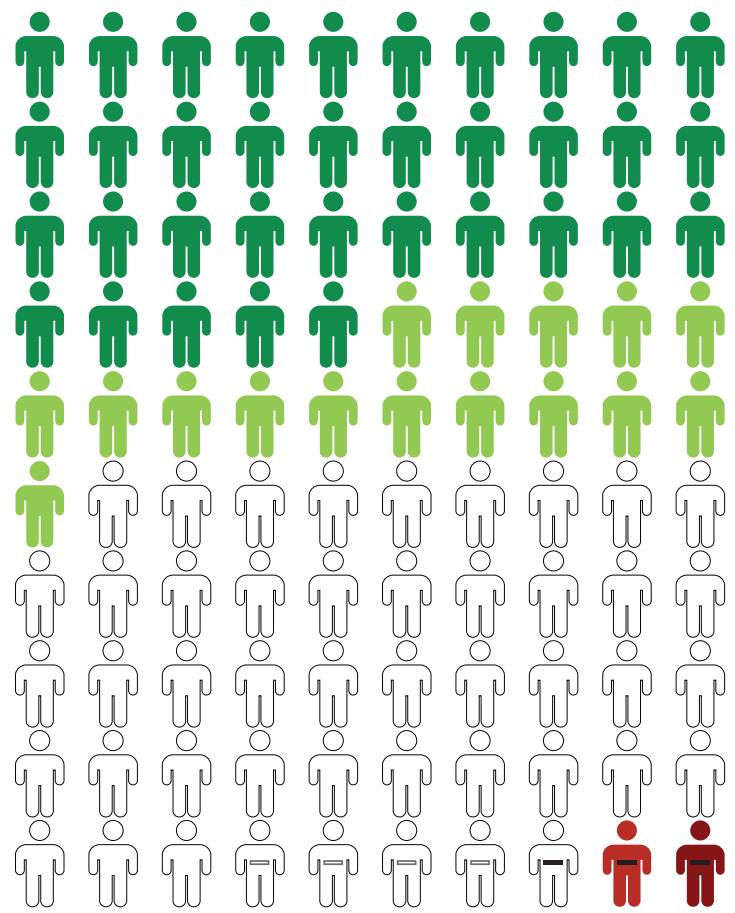

Outcome changes following treatment:

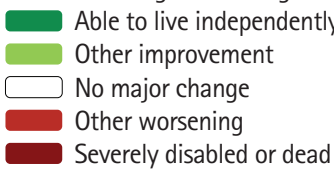

Thrombectomy for late, imaging selected

AIS due to large vessel occlusion: DEFUSE 3 Trial (based on 6-level mRS disability scale)

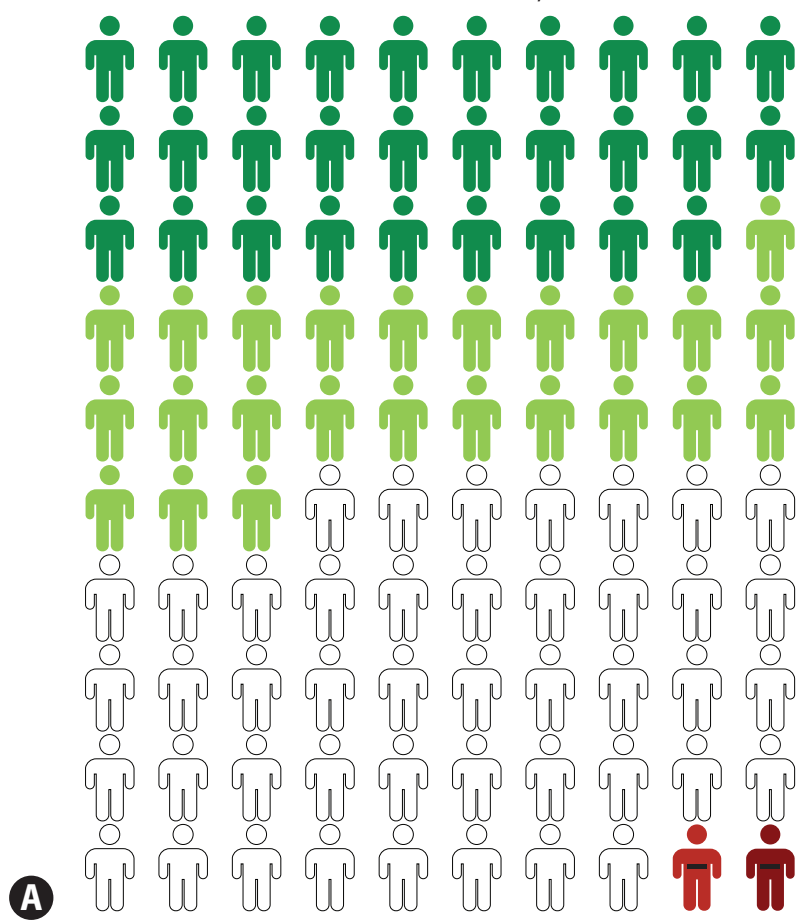

B

$\square$ New territory infarct

Early worsening with brain bleeding

Supplementary Figure 2. Choice Consequence Matrix type visual decision aid depicting the benefits and risks of endovascular thrombectomy (EVT) in latepresenting, imaging-selected patients selected in the DAWN (Clinical Mismatch in the Triage of Wake Up and Late Presenting Strokes Undergoing Neurointervention With Trevo) trial (A) and the DEFUSE 3 (Endovascular Therapy Following Imaging Evaluation for Ischemic Stroke 3) trial (B). Colors indicate changes in 3-month level of global disability that occur as a result of EVT, considering 6 levels of the modified Rankin Scale (mRS). Dark green, attainment of functional independence (mRS 0-2) as a result of thrombectomy; light green, improved disability outcome (other than functional independence) as a result of thrombectomy; dark red, severely disabled, or dead outcome (mRS 5-6) as a result of thrombectomy; light red, worse disability outcome (other than severely disabled/ dead) as a result of thrombectomy; closed dash, symptomatic intracranial hemorrhage as a result of thrombectomy; and open dash, new territory infarct as a result of thrombectomy (only reported for DAWN trial). Figure freely available under a Creative Commons 4.0, use freely with attribution license. AIS, acute ischemic stroke. 
Medical management for late, imaging selected AIS due to large vessel occlusion

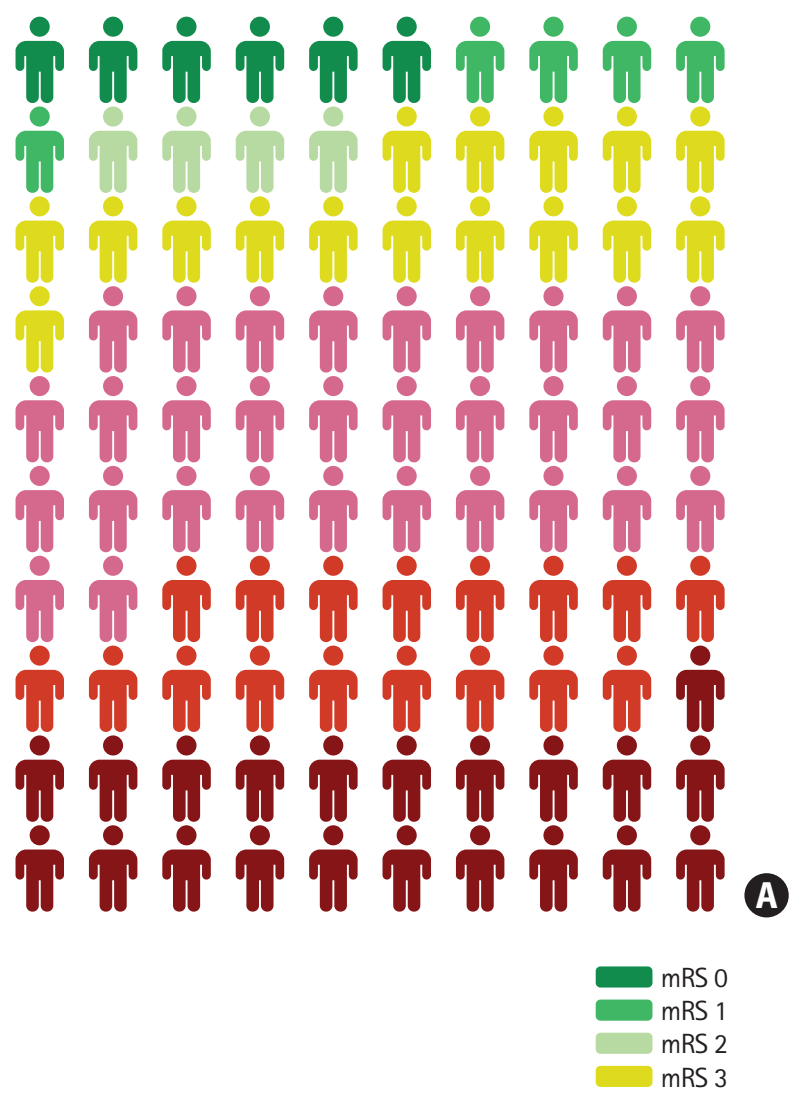

Thrombectomy for late, imaging selected AIS due to large vessel occlusion

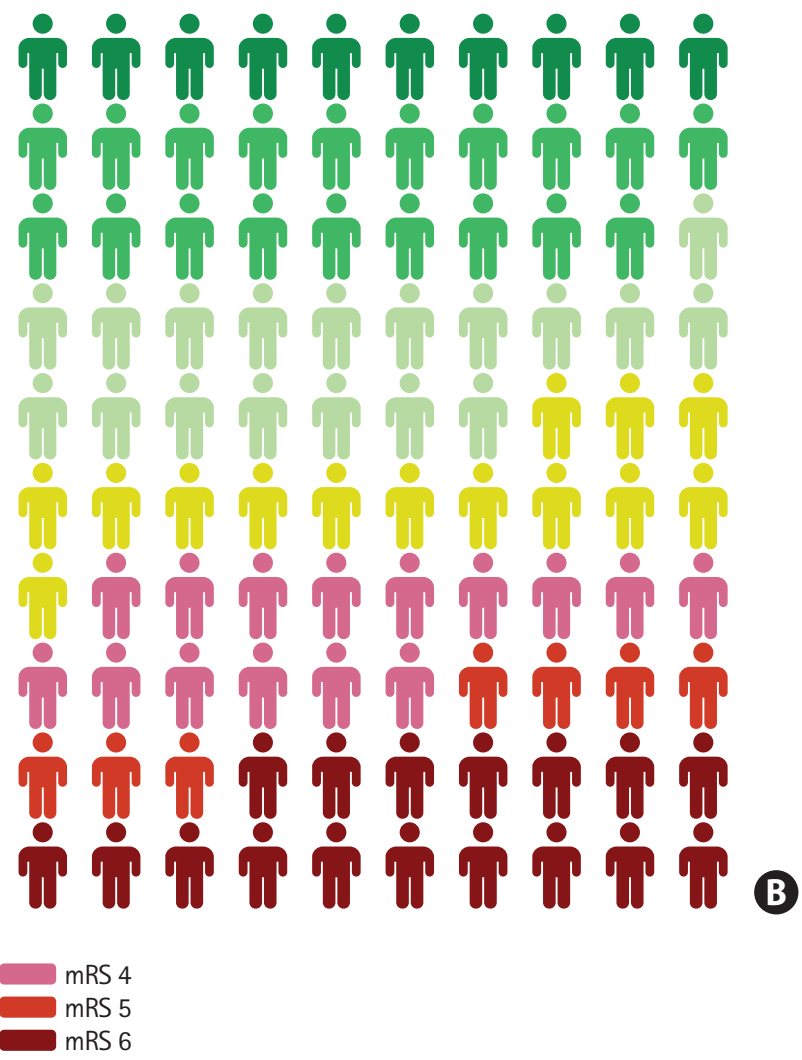

Supplementary Figure 3. Choice Comparison type visual decision aid depicting modified Rankin Scale (mRS) disability level outcomes expected in late-presenting, imaging-selected patients when treated with medical management alone (A) vs. endovascular thrombectomy+medical management therapy (B), based on pooled DAWN (Clinical Mismatch in the Triage of Wake Up and Late Presenting Strokes Undergoing Neurointervention With Trevo)-DEFUSE 3 (Endovascular Therapy Following Imaging Evaluation for Ischemic Stroke 3) data. Figure freely available under a Creative Commons 4.0, use freely with attribution license. AIS, acute ischemic stroke. 